\title{
A Novel Sintered Porous Micromixer for the Effective Mixing of Biologics and Scale Model Investigation of Micromixing Mechanisms
}

\author{
J. Stevens", G. Brossard, A. Blom, A. Douteur and Y. Delmotte \\ Bio Surgery Exploratory Group, Baxter R\&D Europe, Alliance Park, Braine-l'Alleud, Belgium
}

†Corresponding Author Email: justintstevens@gmail.com

(Received February 7, 2011; accepted June 19, 2011)

\begin{abstract}
This paper presents a parametric investigation of a porous 3D micro-mixer as well as a 10:1 scale model of the same used to examine the influence of the contributions of both diffusion and advection to successful mixing of fluids of different viscosity. Experiments at both scales implement the laser induced fluorescence technique to capture the evolution of concentration gradients at the mixer outlet. Mixing performance strongly increases with flow rate in the micromixing apparatus but only moderately in the scale-up suggesting important scale-dependent manipulation of diffusion.
\end{abstract}

Keywords: Mixing, Micromixer, Porous media, Chaotic advection

\section{INTRODUCTION}

The field of microfluidics has exploded in the last several decades. Within this exciting and relatively new frontier, one area of particularly interesting and rapid development has been that of micro-mixers, mixing devices whose flow-paths are on the order of tens or hundreds of microns. Mixing has traditionally been intimately connected with turbulence and the stretching and folding of fluid interface Ottino et al. (1990), however on the micro-scale the general lack of turbulence due to low Reynolds number flows makes mixing a challenge. In addition to small Reynolds numbers, which characterize the relative importance of fluid inertial forces in relation to viscous forces, the physics associated with surface forces tend to dominate over body forces largely due to the increased surface to volume ratios in comparison to their macro counterparts Ho et al. (1998). In these regimes, mixing is dependent on the slow process of molecular diffusion and mixers must be engineered to exploit the deviation from the familiar physics that dictate macro-scale flows Beebe et al. (2002). The clever manipulation of the physics controlling microfluidic flows has brought about the development of a huge number of novel micromixing devices; so many that they have been listed in tables in review papers, categorized by what basic physical forces they manipulate to enhance mixing Jayaraj et al. (2007). Micromixers are generally classified as active or passive mixers; active mixers use an external source of energy to speed up the diffusion-dependent mixing process whereas passive mixers depend only on the mixer geometry and the energy driving the fluid flow Nguyen et al. (2004). The ability of passive mixers to improve mixing on the micro scale without the costly addition of an external energy source as well as manufacturability reasons, make them a desirable option for their integration into industry; an issue that has been considered by microfluidics experts to be of paramount importance for the field to reach its full potential Whitesides (2006).

A host of different passive mixers have been generated in just the last ten years and new novel geometries are continuously being developed and reported. Further classification of passive mixers can be made by categorizing them according to what strategies they employ to enhance mixing. The most common approaches include fluid lamination, split and recombine methods and chaotic advection Chew et al. (2005). The fluid lamination approach arises from the universal goal of micromixing: to reduce the length scale over which diffusion acts. This approach has been used to physically separate the fluid streams into smaller segments such that the striation thickness is reduced Bessoth et al. (1999), thus exploiting the square root dependence of the phenomenon Munson et al. (2003). Because this approach can be implemented to successfully mix fluids without the need to increase Reynolds number, it has been noted to be particularly well suited for viscous flows such as polymer melts Schonfeld et al. (2004).

The split and recombine method as its name indicates, focuses on splitting the fluid flows into smaller streams and then recombining them such that the surface or contact area between the two fluids is increased and thus the distance over which diffusion must act is reduced. This technique has been demonstrated with 
both repeating geometries interrupting the flow-path (Bertsch et al. 2001; Park et al. 2009) as well as randomly distributed flow-splitting geometries (Wang and Li 2007; Rohr et al. 2001; Mair et al. 2009). One design implements both of these concepts using a lattice system that is homogeneous on the micro-scale, yet each individual lattice component is composed of randomly distributed silica nano-spheres Xiao et al. (2010)

The randomly distributed flow-splitting geometries such as those found in porous media, under the appropriate circumstances also have the ability to stretch and fold the flows, further improving mixing, which is related to one of the more interesting mechanisms by which mixing is undertaken, chaotic advection. The term "chaotic advection" was first introduced in the early eighties as a proposed name for a regime in which random passive tracer motion can be found in a laminar velocity field Aref (1984). Since its initial demonstration in an idealized 2D unsteady flow, chaotic advection has been shown to be present in three-dimensional steady flows provided the geometry is of sufficient complexity Cartwright et al. (1999). One of the first realizations of chaotic advection in a microchannel was the so-called "herringbone mixer", which in addition to reporting a short mixing length, demonstrated that the device was able to generate transverse flows in a micro-channel. This trait was found in other work to improve micro-mixing Johnson et al. (2002) and was noted as being responsible for the stretching and folding of the flows and is accredited with making it "possible to design steady chaotic flows for use in microfluidics systems" Stroock et al. (2002). Chaotic advection is a popular approach to micromixing due to its contribution from both diffusion and fluid advection and is reported in a number of papers (Simonnet et al. 2005; Liu et al. 2000; Therriault et al. 2003). Although it is possible to realize chaotic advection in very low Reynolds number systems, mixing generally improves with increasing Reynolds number Chew et al. (2005). One particularly novel design combines several concepts, operating predominantly in the diffusion regime with split and combine action for lower flow rates, but then relies almost exclusively on chaotic advection for higher flow-rates Hong et al. (2004). Literature suggests that complicated geometries with tortuous and non-periodic flow paths are good breeding grounds for chaotic advection (Cartwright et al. 1999; Ottino et al. 1990; Therriault et al. 2003 ); however the manufacturing processes required generally make large scale production difficult, a critical step for integration into industry.

In response to a mixing need at Baxter Healthcare Corporation, a series of sintered porous discs were investigated for potential use as micromixers for mixing Fibrinogen and Thrombin in a medical device. A sintered polyethylene disc now known as "Vyon F" has been identified as a novel effective micro-mixer for this task. The non-Newtonian nature of these biological fluids as well as a viscosity difference of two orders of magnitude, contributes to the difficulty of this mixing problem. Despite these challenges, the tortuous and randomly distributed flow paths provided by the sintering process encourages transverse motion which is expected to facilitate chaotic advection in a manner reminiscent to the twisted pipe scenario Jones et al. (1989) and thus improve the effectiveness of diffusion by allowing it to operate on a more appropriate length scale. The unique geometry of this device was studied and characterized in detail using a high resolution tomographic scanner. Both tortuosity as well as anisotropy of the fluid paths were characterized and provide some insight as to the success of the mixer. In addition to the extensive characterization of the geometry of this novel device, a parametric study involving two scales of the system was conducted to both demonstrate the ability of the device to mix fluids with dissimilar viscosities (as is the case with Fibrinogen and Thrombin) at low Reynolds numbers as well as to better understand the individual contributions of diffusion and transverse motion to effective mixing demonstrated by this device.

\section{EXPERIMENTAL SET-UP AND Procedure}

\subsection{Porous Media Structure}

The micro mixer presented in this work is a sintered porous polyethylene disc $(\varnothing 3.8 \times 1.5 \mathrm{~mm})$ with a porosity of $38.9 \%$ and an average pore size distribution of $60 \mu \mathrm{m}$ produced by Porvair (UK). Early testing showed that the mixing disc significantly outperformed packed beds of uniform spheres even when both systems had the same permeability. This led to curiosity about the geometry of the disc, specifically to how and why the sintered geometry improved mixing over a packed bed. Before Vyon F was decided upon as the ideal candidate for the Fibrinogen-Thrombin mixing problem, other types of porous media were considered and their geometry analyzed. An in-depth study of the geometries was conducted via a high-resolution $(1.4 \mu \mathrm{m}$ per layer) topographic scan of each disc (courtesy of J. Vicente, Marseille, France).

All of the geometries investigated were made from irregular shaped pellets of various sizes that once sintered, encourage tortuous paths resulting in an easily manufactured yet very complex 3D flow path which creates an environment favorable for chaotic mixing. The scans of the samples were used to build CAD models of each media and were statistically analyzed and characterized in several categories of interest with special focus on tortuosity and average pore size distribution, both of which were shown to be related to the initial pellet size and shape. The starting grain before the sintering process has a direct effect on both the tortuosity as well as the average pore size in the porous media. These two geometry factors both have an important impact on the quality of mixing that will take place in the disc. A higher level of tortuosity has a tendency to increase the degree of transverse motion which is beneficial for mixing, however the smaller the pore size, the more effective diffusion becomes. The statistical characterization of these geometries highlighted a trade-off in the question of tortuosity vs. average pore size when analyzing the distribution of tortuous paths in the discs. The results for the simulations showed that larger the size of the starting grain, the higher the tortuosity was, however there was 
also a higher level of variation in tortuosity in the media which in turn led to heterogeneous transverse motion and inferior mixing despite higher average tortuosity.

The top performing mixing disc, Vyon F, boasted a balance of these traits. The experimental testing presented in this paper focuses on the results of this most successful mixing disc, Vyon $\mathrm{F}$, and attempts to connect important factors in its geometry as well as the evolution of concentration gradients in the mixing tests to shed light on why this mixer is successful where the other candidates were not.

\subsection{Description of the System}

The study investigated the effect of Reynolds number on the ability to mix two fluids of different viscosity (0.001 Pa.s and 0.1 Pass respectively) in both the $\varnothing 3.8$ $\mathrm{mm}$ and $1.5 \mathrm{~mm}$ thick disc and a scale-up model. The CAD model of the geometry was used to create an 11.5:1 scale-up model of the Vyon F disc, realized via stereo lithography (SLA) (Materialize, Leuven, Belgium) (Fig. 1). The odd scale was chosen instead of 10:1 due to limitations in SLA resolution. Mixing performance was evaluated for the same range of Reynolds numbers at both the scale-one and the scaleup (table 1) models.

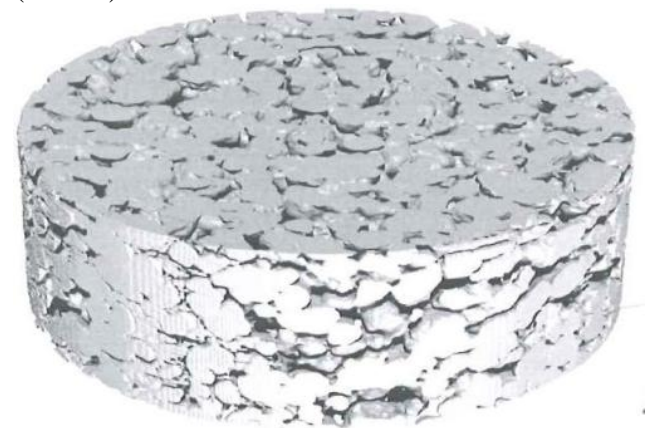

Fig. 1: CAD Model Generated from Scan of Mixingdisc

\subsection{Brief Dimensional Analysis for Scaling}

The Reynolds number is an important dimensionless value in this system that compares the impact of the inertia of the fluid to the viscous effects. Reynolds number is calculated differently depending on the shape of the flow path. Due to the two different viscosities of the fluids being mixed in this system as well as the irregular complex geometry, an exact Reynolds number calculation for the fluid inside the actual porous media cannot be confidently reported. Thus to match flow conditions between the two scales in this parametric study, Reynolds number is calculated at the fluid inlets going into the test models for both water and glycerolwater and is approximated as:

$\operatorname{Re}=\left(\frac{\rho Q d}{\mu A}\right)$

Reynolds number is respected during scaling, the density $\rho$ and viscosity $\mu$ will remain constant as the scale increases, however characteristic length $d$ as well as cross sectional area $A$ will inherently increase proportional to the scaling factor. Thus for the scale-up apparatus, if the flow rate $Q$ is scaled with the scaling factor $(11.5: 1)$ and the SLA model respects the micromixer geometry, the system should exhibit similar behavior to the micro-model with regard to fluid inertial and viscous effects. Reynolds number can also be approximated for a packed bed by the equation:

$\operatorname{Re}=\left(\frac{\rho Q D_{\text {sphere }}}{\mu A(1-\phi)}\right)$

Where $D_{\text {sphere }}$ is the average sphere size used to form the packed bed and $\varphi$ is the porosity of the packed bed. This value however, may not be completely accurate for our porous media because the major differences in geometry between a packed bed and this media, it will however be included to demonstrate that inertial effects are drastically diminished in the presence of porous media.

The Peclet number is another important dimensionless number and is used to describe the relation between the impact of fluid advection and diffusion in the system and is defined as:

$P e=\left(\frac{L v}{D}\right)$

Where $L$ is the characteristic length, $v$ is the superficial velocity and $D$ is the mass diffusion coefficient. Peclet number is also respected with Reynolds number in each scale tested.

Table 1 Dimensionless Numbers and Associated Flow Rates

\begin{tabular}{|c|c|c|c|c|}
\hline \multicolumn{5}{|c|}{ Flow Rate in $\mathrm{mL} / \mathrm{min}$} \\
\hline Scale 1 & 2 & 6 & 20 & 35 \\
\hline Scale-up & 23 & 69 & 230 & 405 \\
\hline \multicolumn{5}{|c|}{ Reynolds Numbers Calculations for Water } \\
\hline $\operatorname{Re}_{\text {Fluid Inlet }}$ & 49 & 146 & 488 & 854 \\
\hline $\operatorname{Re}_{\text {packed bed }}$ & 0.7 & 2.2 & 7.2 & 12.6 \\
\hline \multicolumn{5}{|c|}{ Reynolds Number Calculations for Glycerol-Water } \\
\hline $\operatorname{Re}_{\text {Fluid Inlet }}$ & 0.6 & 1.8 & 6.0 & 10.5 \\
\hline $\mathrm{Re}_{\text {packed bed }}$ & 0.009 & 0.027 & 0.089 & 0.155 \\
\hline \multicolumn{5}{|c|}{ Peclet Number in Average-Sized Pore } \\
\hline $\mathrm{Pe}_{\text {Average Pore }}$ & 1176 & 3527 & 11757 & 20574 \\
\hline
\end{tabular}

\subsection{Planar Laser Induced Fluorescence (PLIF)}

Flow visualization is a valuable mixing analysis tool and has been able to provide qualitative mixing efficiency information in the past by analyzing the color variation in dyed liquids by extracting RGB values from digital images Schonfeld et al. 2004; Bertsch et al. 2001; Wang et al. 2007; Xiao et al. 2010; Hong et al. (2004). Concentration gradients on the other hand, can be used to gain quantitative information about the mixing by relating experimental grayscale intensity variations from ph-based reactions (Munson et al. 2003; Liu et al. 2000) or fluorescently tagged fluids Bessoth et al. (1999); Park et al. 2009; Stroock et al. 2002; 
Simonnet and Groiseman 2005; Therriault et al. 2003) to calibration curves where grayscale camera intensity values (from CCD cameras) are related to known dye concentrations in a solute and thus experimental images can be iteratively "corrected" by a correction factor which translates the grayscale values in an image to the concentration or presence of fluorescent dye in an observation area. In this experiment the planar laser induced fluorescence (PLIF) technique (Mair et al. 2009; Pust et al. 2006) is implemented because of its ability to isolate and digitally capture cross-sections of geometry with minimal influence from out-of-plane fluid mixing. A Rhodamine $6 \mathrm{G}\left(\lambda_{\text {excitation }}=532 \mathrm{~nm}\right.$; Aldrich) aqueous solution is used $\left(4 \times 10^{-6} \mathrm{M}\right)$ for the fluorescently tagged fluid (the PLIF technique measures the presence of the fluid tagged with fluorescent dye in the cross-sections captured on camera) and a $0.1 \mathrm{~Pa} \cdot \mathrm{s}$ (85\% glycerol-water solution, Merck) for the untagged fluid. The $4 \times 10^{-6} \mathrm{M}$ concentration of Rhodamine dye in water is taken to be the full or $100 \%$ concentration of dye in the system for the remainder of this paper. The relatively large difference in viscosity was characteristic of the unique biologics mixing problem at hand and was respected.

\subsection{Experimental Apparatus}

The use of PLIF demands optical accessibility to the flow path which was provided by the assembly of various multi-component PMMA housings. A schematic of the entire experimental apparatus for the lateral investigations is shown in Fig. 2.

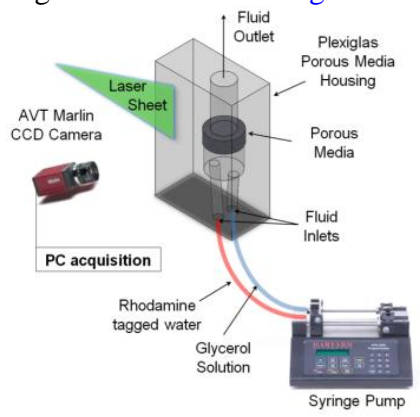

Fig. 2. Experimental Set up for Lateral Investigations

The interior flow domain of the housings consist of three main parts, a Y junction bringing the two fluids to the disc, a chamber to house the disc and a constant diameter pipe where the concentration gradients are captured. One model was built allowing the lateral cross section of the pipe to be captured and one allowing observation of the circular cross section (Fig. 3). Fluids were pumped into the underside of the porous media housing by a Harvard PHD 2000 syringe pump.

For each of the two model housings built, a laser sheet $(\lambda=532 \mathrm{~nm})$ illuminated the plane of interest, causing the dye in the tagged fluid to fluoresce. A CCD grey-scale camera (Marlin, AVT) equipped with a narrow bandpass filter (568 $\pm 10 \mathrm{~nm}$, Edmund Optics) captured a 15 fps development of the dye concentration gradients at different Reynolds numbers.

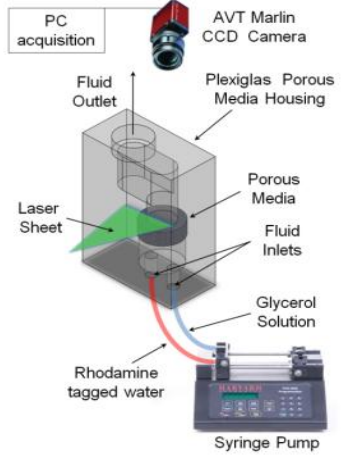

Fig. 3. Experimental Set-up for Circular Cross-section

Geometrically analogous model-housings were constructed for both scales of this apparatus and the same testing procedure at the same Reynolds numbers was performed, three test runs were completed for each Reynolds number at each scale.

\subsection{Flow visualization experiments}

Flow visualization experiments were conducted in both the lateral and circular cross-sectional housings for both scales to examine the quality of mixing over the range of Reynolds numbers listed in Table 1 . The flow rates used in the scale-one apparatus were 2, 6, 20 and 35 $\mathrm{mL} / \mathrm{min}$, analogous flow rates used in the scale-up were 23, 69, 230, $405 \mathrm{ml} / \mathrm{min}$, respecting Reynolds number.

Video was taken at $15 \mathrm{fps}$ as soon as the fluid began to be pumped and continued for approximately 10 seconds after steady-state behavior was reached. The video was saved as an 8-bit grayscale series of images for convenience in post processing. Control experiments were also conducted with the mixing disc absent from model-housings for comparison. Post processing of the experimental images allowed analysis of the degree to which the different flow rates affected fluid mixing for both scales.

For the lateral cross-section investigations, the laser sheet was placed vertically along the center of the diameter of the pipe illuminating the plane perpendicular to the camera as in (a.) in Fig. 4 the view the camera then captures is detailed in (b.). In postprocessing, the area of interest is cropped and calibrated for a final image (c.); this data will be introduced in Figs. 9 and 10 in the results and discussion section.

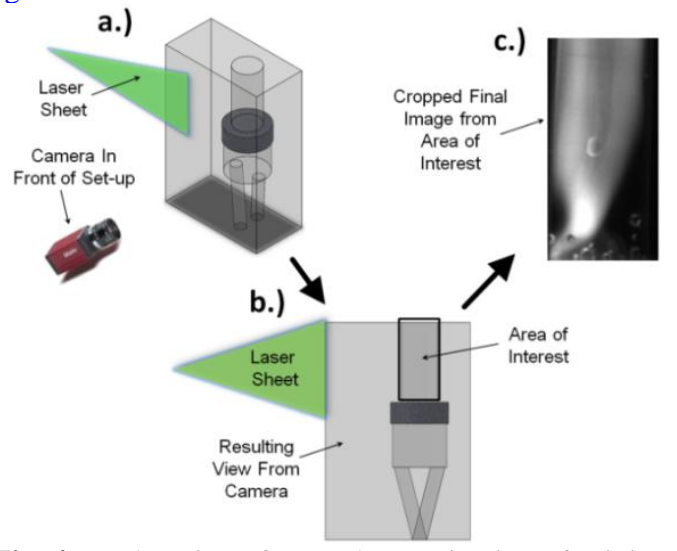

Fig. 4. Explanation of Lateral Investigation of Mixing Efficiency 
The circular cross-section tests illuminated a section of the pipe $0.5 \mathrm{~cm}$ after the disc and placed the camera above the test set-up such that the camera looked down into the pipe as in (a.) in Fig. 5. The resulting camera view shows a circular cross section of the pipe (b.). Any area of the image other than the cross section is masked in post processing for clarity; data from this view is also included in Figs. 9 and 10 along with the lateral investigation images in the results and discussion section of this paper.
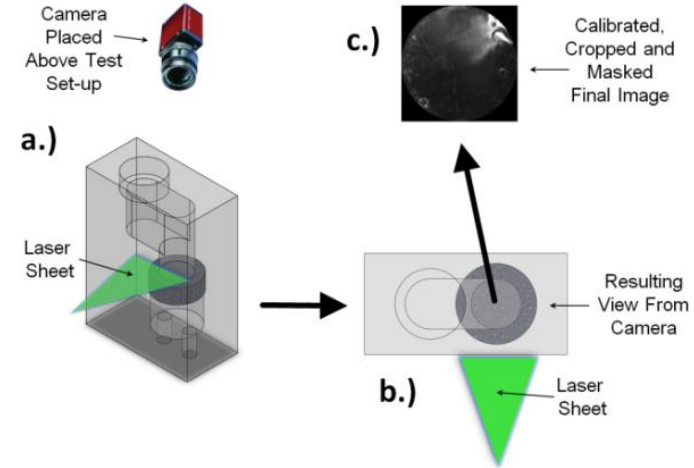

Fig. 5. Explanation of Top Cross-section Investigation of Mixing Efficiency

\subsection{Mixing Evaluation Post-Processing}

Analysis of mixing performance for each system was performed by post-processing the flow visualization images in Matlab (2010a). An in-house code was developed to account for inhomogeneous illumination by the laser and calibrate each image in the video series using an eleven point calibration curve. The calibration procedure correlates the gray scale intensity captured by the CCD camera sensing the dye fluorescing to Rhodamine dye concentration. This is done by capturing images of the flow domain filled with homogeneous concentrations of dyed-water, ranging from 0 to $100 \%$ in increments of $10 \%$. Inhomogeneous illumination (the flow domain was more brightly illuminated on the side of the laser) is accounted for by normalizing the image series by the $100 \%$ dye image (again " $100 \%$ dye" means an undiluted $4 \times 10^{-6} \mathrm{M}$ concentration of the Rhodamine dye in the water). Since the camera was fixed for each test run, a single pixel can be tracked through a series of images and assumed to represent the same location in the observation area (upper image in Fig. 6). Each pixel is then assigned a unique quadratic equation that describes the camera intensity vs. dye concentration for that pixel through the 11 image series (lower plot in Fig. 6). It is this quadratic equation that converts a grayscale intensity value to a percent presence of Rhodamine dye value.

For example, if a pixel has a grayscale value of 100 it is normalized by its corresponding pixel in the $100 \%$ dye calibration image. For the sake of the example, let us say that the corresponding pixel in the $100 \%$ dye calibration image is 245 out of a possible 256 , the normalized value for that pixel would be 0.408 (the normalization of 100/245). This value is then substituted into the quadratic calibration equation assigned to that pixel (Fig. 3) which would then be solved by the Matlab program and yield a percent dye value for that pixel of approximately $30 \%$ (see plot in Fig. 3). The normalization of the images by the $100 \%$ dye (full concentration of $4 \times 10^{-6} \mathrm{M}$ dye in water) has the additional benefit of correcting heterogeneous lighting effects.
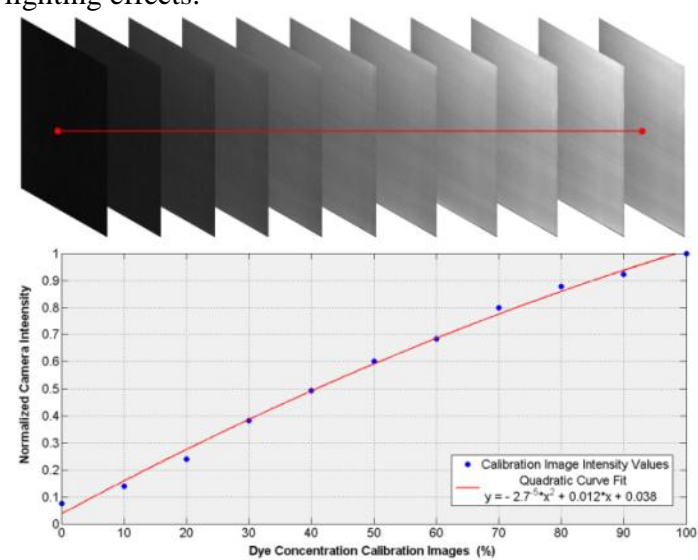

Fig. 6. Calibration Procedure Example for one Pixel

Results from the control test indicated that on the timescale of the observation, the diffusion of the Rhodamine dye into the glycerol-water solution is negligible (Fig. 7) and thus 50\% presence of dye (equal portions of non-dyed glycerol and the full concentration of the dyed-water) is taken to be analogous with ideal mixing.
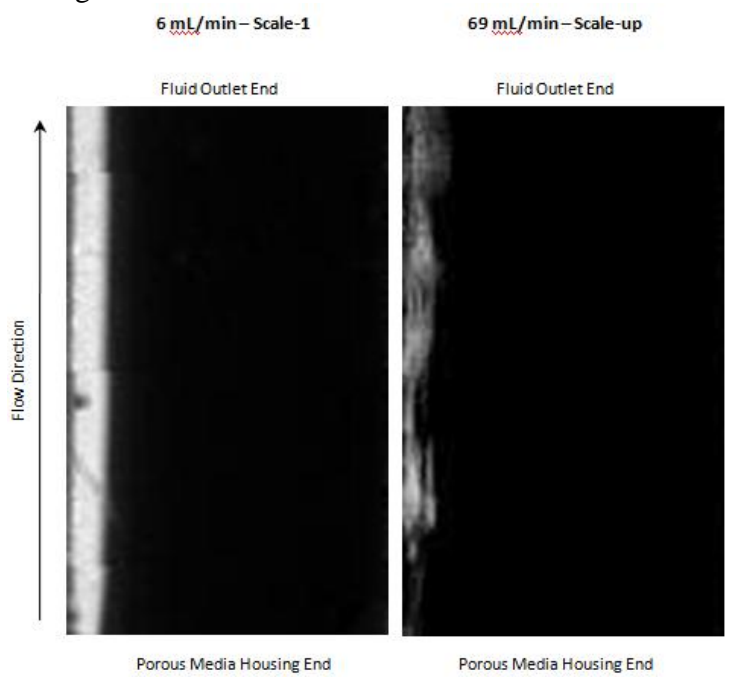

Fig. 7. Scale Model Control Comparison

Evaluation of mixing in literature has been both qualitatively and quantitatively in many ways. Various units have been developed to gauge mixing, such as the mixing moment Simonnet and Groiseman (2005), mixing index Wang and $\mathrm{Li}$ (2007), deviation factor Liu et al. (2000), mixing efficiency (Park et al. 2009; Xiao et al. 2010) or in cases of entire cross-sections (as opposed to a single concentration vector) a coefficient of variation (COV) has been calculated for the area Pust et al. (2006).

However since the presence of dye has been taken to represent mixing, an aesthetically pleasing combination of qualitative color schemes and quantitative concentration values lends itself in the $3 \mathrm{D}$ contour plot (Fig. 8). The left portion of Fig. 8 shows the experimental top cross section image from which the 
3D contour plot is constructed. The right side of the figure shows the plot, where the $\mathrm{x}$ and $\mathrm{y}$ axes represent the area of the experimental image and the vertical $\mathrm{z}$ axis represents the presence of dye in the cross-section. This is a valuable representation of the data because it allows both a qualitative viewing of the data with color, but also quantitative information because of the calibration procedure translating intensity values to concentration values. This plot is used in the analysis in the following section.

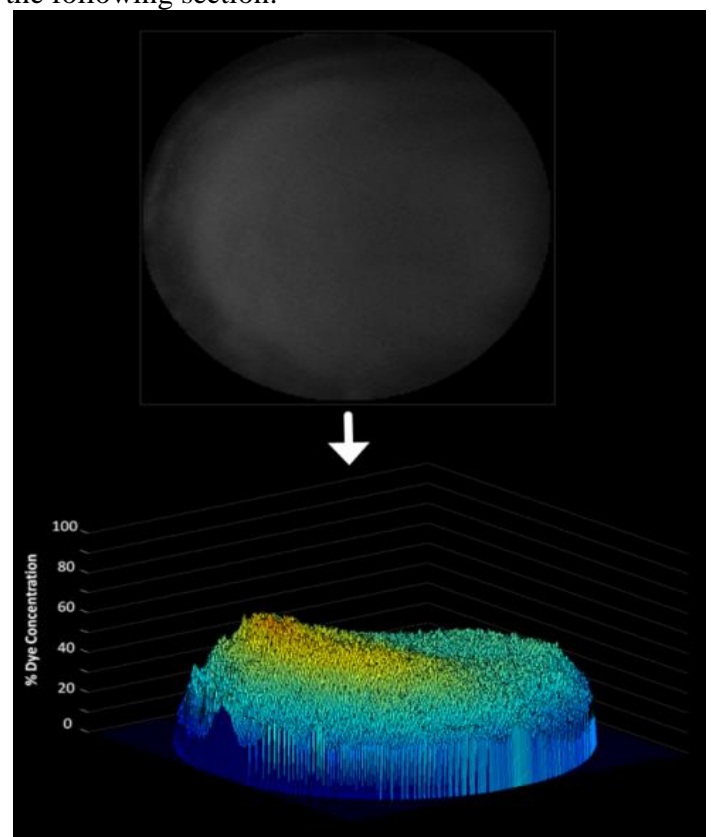

Fig. 8. 3D Contour Plot of Dye Presence in Pipe Top Cross Section

\section{RESUlTS AND DISCUSSION}

Operating conditions of micromixers are often characterized by their relevant dimensionless numbers. One useful example of this is the Peclet-Reynolds (PeRe) number diagram Nguyen et al. (2004). This logarithmically scaled diagram uses the Reynolds and Peclet numbers that the devices operate at to group them into categories of similar operating principles. The sintered mixing-disc fits into the "passive mixing at medium Reynolds number" group along with two other very successful mixing devices, the modified Tesla structure Hong et al. (2004) and the 3D serpentine channel Liu et al. (2000).

According to this placement on the Pe-Re diagram, as well as the tortuous nature of the sintered geometry, it is likely that this device exhibits chaotic advection. This is supported by the flow visualization evidence, where mixing is dramatically improved as the flow-rate increases, a characteristic of mixers that utilize chaotic advection in these operating conditions.In an effort to digest the large quantity of data collected in this parametric study investigating the effect of mixing quality on flow rate, the plots in Fig. 9 and 10 attempt to provide a representative image for both the lateral and top cross section investigations at each flow rate tested for comparison. The top cross section views (Fig. 5 ) appear in the first row of plots in the figure while the lateral cross section (Fig. 4) plots appear in the second row. The third and final row provides a plot of the concentration across the diameter of the lateral cross section just after the porous media and is included as a means to represent the mixing quality without using the experimental images.

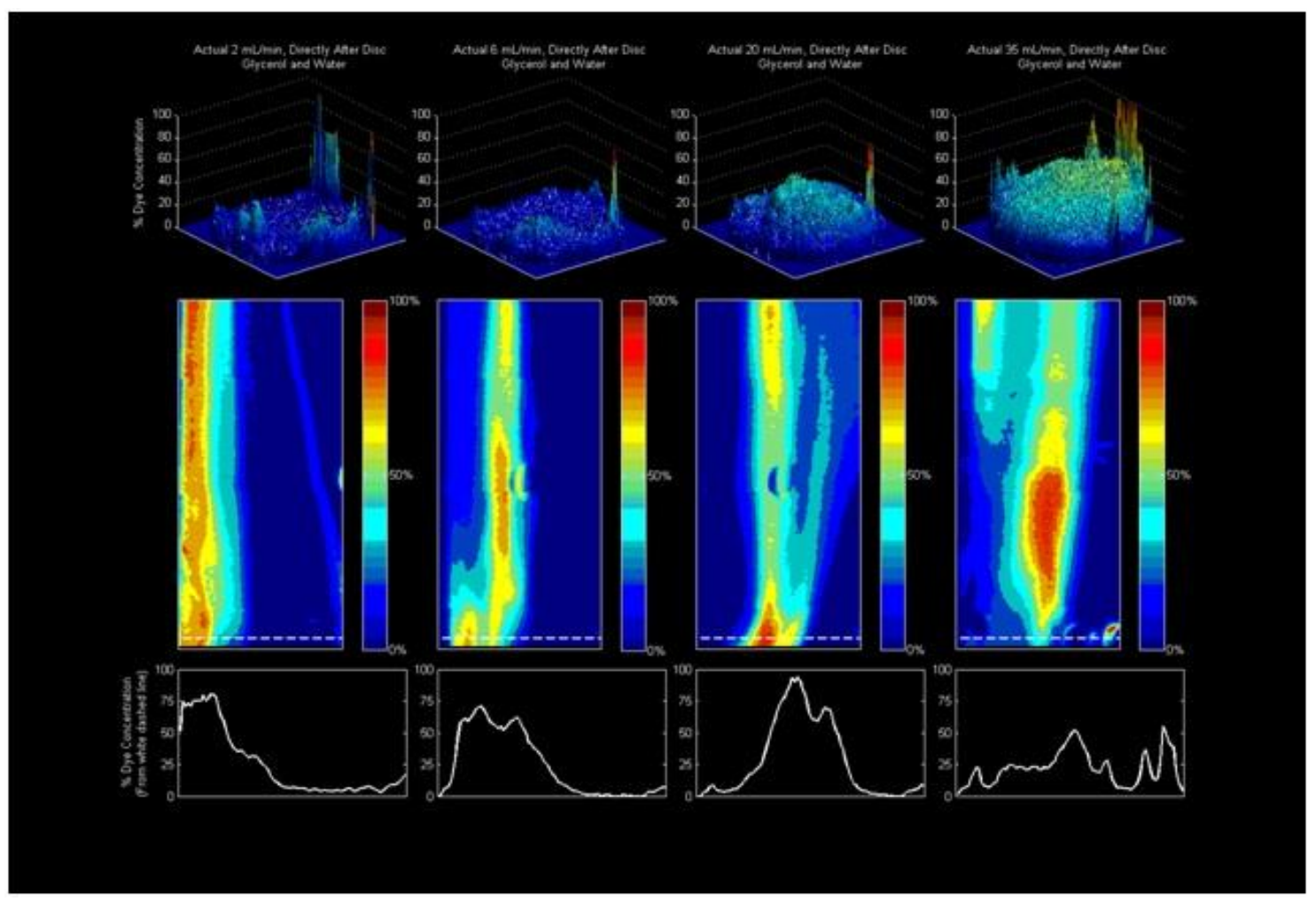

Fig. 9. Effect of Flow-rate on Mixing Quality at the Outlet of the Micromixer 
In addition to the general problem of difficulty mixing at low Reynolds, the task is further complicated by a difference in the density between the two fluids which causes the less dense fluid to be pressed against the wall of the flow path. This can be clearly seen in the control images in Fig. 7 where the denser glycerol has a tendency to force the less dense water to the walls of the flow path. This makes the lateral transport difficult because the water has to fight against the density effects of the glycerol as it travels through and after the disc. For this reason, the large degree of lateral transport in the scale- 1 model shown in Fig. 9 is impressive. The degree to which mixing is improved with flow rate is significant and easily appreciated in the 3D contour plots in the top row of Fig. 9. Another interesting trend that demands attention is the migration of the dye towards the center of the chamber and its subsequent improved dispersion with flow rate, as characterized by the concentration profiles (which are representative of the white dashed line in the center contour plots) found in the bottom row of Fig. 9. The improved lateral transport with increasing Reynolds number for devices in this group of operating conditions (similar Pe and $\mathrm{Re}$ ) is consistent with literature (Nguyen et al. (2004); Chew et al. (2005); Liu et al. (2000); Hong et al. (2004); Vijayendran et al.).

Despite the drastic mixing quality improvements seen in the micromixing device, only minor improvements are observed in the scale-up, where obvious evidence of improved lateral transport does not appear until a flowrate analogous to $35 \mathrm{ml} . \mathrm{min}$ (Fig. 10). This suggests that although geometry is an important factor for improved mixing, the key seems to lie in the combination of geometry and a scale that can maximize the effectiveness of diffusion over the fluids journey both through and after the mixing device. This can be demonstrated with an example of how length scale impacts diffusion time.

Using a general model for diffusion of $l^{2}=2 D t$ (3) where $l$ is the length over which diffusion must occur, $D$ is the diffusion coefficient and $t$ is the time necessary for the diffusion to occur. Let the diffusion coefficient for the water and the glycerol aqueous solution be approximately $1.5 \times 10^{-10} \mathrm{~m}^{2} / \mathrm{s}$ Balasubramaniam et al. (2002). Diffusion across the average pore throat $(600$ $\mu \mathrm{m})$ in the SLA mixing disc under these conditions would take about 20 minutes. In contrast, the same diffusion time in the micromixer, unassisted by advection would take 12 only seconds. If secondary flows develop inside these tortuous paths that exponentially stretch and fold fluid interfaces Therriault et al. 2003, then its combined effect with diffusion could have a much larger impact. If the striation thickness was for example reduced to say $10 \mu \mathrm{m}$ then diffusion would only take one third of a second to finish the job.

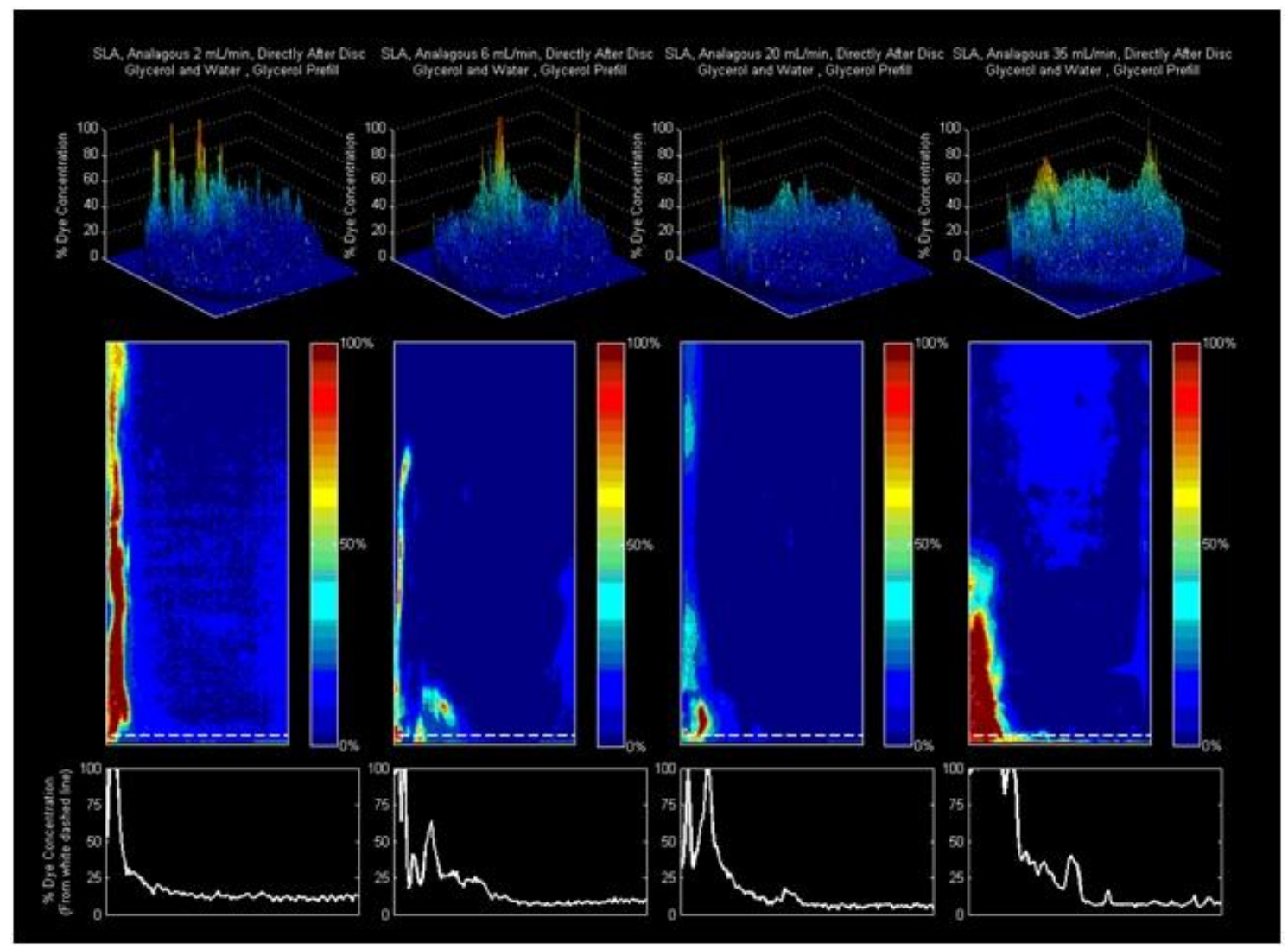

Fig. 10. Effect of Flow-rate on Mixing Quality at the Outlet of the Scale-up Mixing-Disc

There is however a tradeoff between the flow rate increase and the time diffusion is able to work inside the This amount of time would require a striation thickness of only $6 \mu \mathrm{m}$ for ideal mixing to be reached. Although a quantitative assessment of the distance between fluid interfaces is not available in this work, 
the resulting concentration profiles at the end of the scale-1 observation windows suggest that the mixing disc is rearranging the fluid interfaces in a way that facilitates diffusion to greatly improve the ability of the fluids to mix by the time the end of the observation window is reached, in comparison to the control.

The mixing benefit despite the short time inside the disc also suggests that the degree of lateral transport is related to fluid momentum and once the lateral transport redistributes the fluid inside the disc, diffusion continues the process until reaching the fluid outlet. The evolution of this concentration gradient along the observation window can be observed in Fig. 11 where the lateral view (explanation of lateral view in Fig. 4) of the scale- 1 model has been tested at $35 \mathrm{ml} / \mathrm{min}$.
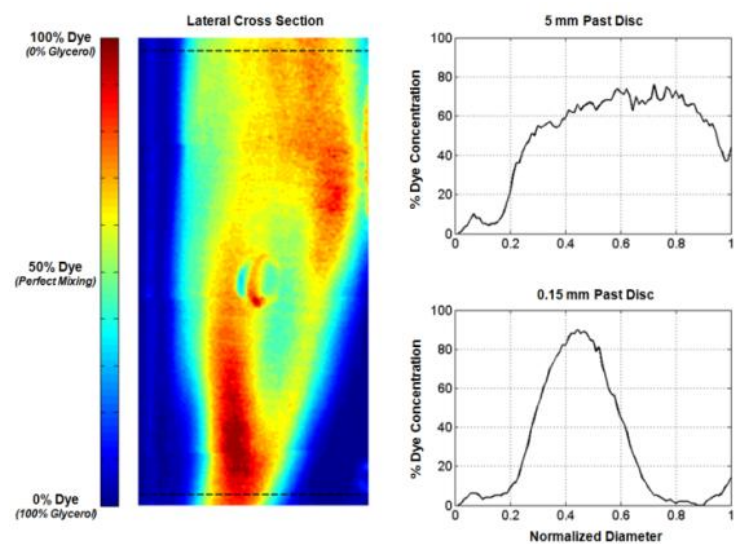

Fig. 11. Concentration Gradients in Scale-1 Model at $35 \mathrm{ml} / \mathrm{min}$

The two concentration plots in the figure monitor the concentration of dye across the diameter both directly after the porous disc (lower plot) and at the end of the observation window furthest away from the porous media (upper plot). The evolution of the concentration gradient from the lower plot to the upper plot shows that there is significant diffusion that improves the overall mixing device, larger the flow rate becomes, the less time the fluid spends inside the device for diffusion to act because of the increased fluid velocity. For example, in the scale- 1 model for a flow-rate of 35 $\mathrm{ml} / \mathrm{min}$, fluid will pass through the disc in 0.0025 seconds. However it will take about 0.25 seconds for the fluid to travel through the disc and to the end of the $5 \mathrm{~mm}$ observation window.

Still other differences in the fluid physics between the two scales could play a role in the different mixing performance. For example, could the increased importance of surface forces perhaps be magnifying the Coanda effect and encouraging a larger amount of lateral motion in micromixer that is not observed in the scale-up? Are the capillary forces in smaller pores acting like pressure activated gates that redirect flow until sufficient pressure is available to overcome the resisting force and pass through them? If so, could this be strategically used in conjunction with flow rate manipulation to redistribute fluid and maximize chaotic advection?

Perhaps the sintering process causes a directional permeability as found in geology, could this coupled with some type of critical Reynolds number trigger a significantly enhanced lateral motion? These are all questions that a better understanding of the system could help to answer and contribute to the optimization of this system as well as its application to other mixing problems.

\section{Conclusions}

The goal of this study was to demonstrate this novel mixing disc's ability to efficiently mix fluids with dissimilar viscosities as well as observe real-time evolution of dye concentration gradients in conjunction with extensive geometric analysis in an effort to better understand the mixing mechanisms that this disc is able to so effectively utilize. Significant flow rate dependence was observed in the micro system but minimal flow-rate impact on the scale-up. This is likely because of differences in the physics related to the difference in the scale of two systems. The improvement observed in the quality of mixing with increased flow-rate in the micromixer is consistent with literature as is the support of complicated geometries in the improvement of mixing (Stone et al. 2001; Andreasen et al. 2008). The complicated 3D geometry of this device was investigated in detail and its favorable balance of small average pore size and large tortuosity caused it to stand out among other porous mixers. The sintered porous disc was demonstrated to be an effective micromixing device for fluids with a difference in viscosity of two orders of magnitude.

The micromixer enjoys a scale in which the advection made possible by the torturous paths (Willingham et al. 2008) created by the sintering process, work together with diffusion to strongly improve mixing efficiency. Additional testing should be done in the future to parametrically investigate the effects of surface tension to better understand the role of capillary effects in the scale-one system. In an effort to understand how fluid momentum affects lateral transport it would be useful to identify a directional permeability value such that the porous media's resistance to flow in both lateral and axial directions could be quantified and related to the tendency to encourage lateral transport.

\section{ACKNOWLEDGEMENTS}

The author would like to thank Michael Bilka and Tyler Zawacki for helpful discussions and direction with regard to the development of the post processing algorithms and Maria Vetrano for her encouragement and support during the early stages of this work. The optically accessible models for the scale-up testing were manufactured at the von Karman Institute for Fluid Dynamics.

\section{REFERENCES}

Andreasen, C.S., A.R. Gersborg, O. Sigmund. 2008. Topology optimization of microfluidic mixers, Int. J. Numer. Meth. Fluids, pp. 498-513.

Aref, H. 1984. Stirring by Chaotic Advection, Journal of Fluid Mechanics, pp. 1-21. 
Balasubramaniam, R., N. Rashidnia 2002. Glenn Research Center at Lewis Field. Diffusivity Measured as a Function of Concentration. [book auth.], Research and Technology. Cleveland: NASA/TM, 2002, pp. 219-220.

Beebe, D.J., G.A. Mensing, G.M. Walker 2002. Physics and Applications of Microfluidics in Biology, Annual Review of Biomedical Engineering, pp. 261-286.

Bertsch, A., S. Heimgartner, P. Coussea, P. Renaud 2001. Static micromixers based on large-scale industrial mixer geometry, Lab on a Chip, pp. 5660.71 .

Bessoth, F.G., A.J. deMello, A. Manz 1999, Microstructure for efficient continuous flow mixing, Analytical Communications, pp. 213-215. 58.

Cartwright, J.H.E., M. Feingold, O. Piro. An Introduction to Chaotic Advection. [book auth.] Villermaux E, Chomez J M, Chate A H. Mixing: Chaos and Turbulence. s.l., Kluwer Academic/Plenum Publishers, 1999, pp. 307-342.

Chew, Y.T., H.M. Xia, C. Shu, S.Y.M Wan. 2005. Techniques to Enhance Fluid Micro-Mixing and Chaotic Micromixers, Modern Physics, Letters B, Vol. 19, pp. 1567-1570.

Ho, C., Y. Tai 1998. Micro-Electro-MechanicalSystems (MEMS) and Fluid Flows, Annual Reviews of Fluid Mechanics, pp. 579-612. 639.

Hong, C., J. Choi, C.H. Ahn 2004. A novel in-plane passive microfluidic mixer with modified Tesla structures, Lab on a Chip, pp. 109-113.

Jayaraj, S., S. Kang and Y.K. Suh 2007. A Review on the Analysis and Experiment of Fluid Flow and Mixing in Micro-Channels, Journal of Mechanical Science and Technology, pp. 536548.

Johnson, T.J., D. Ross, L.E. Locascio 2002. Rapid Microfluidic Mixing, Analytical Chemistry, Vol. 74, pp. 45-51.

Jones, S.W., O.M. Thomas, H. Aref 1989. Chaotic advection by laminar flow in a twisted pipe, Journal of Fluid Mechanics, pp. 335-357.

Liu, R.H., M.A. Stremler, K.V. Sharp, M.G. Olsen, J.G. Santiago, R.J. Adrian, H. Aref, D.J. Beebe 2000. Passive Mixing in a Three-Dimensional Serpentine Microchannel, Journal of Microelectromechanical Systems, Vol. 9, pp. 190197. 501.

Mair, D.A., T.R. Schwei, T.S. Dinio, J.M.J. Frechet 2009. Use of photopatterned porous polymer monoliths as passive micromixers to enhance efficieny of on-chip labeling reactions, Lab on $a$ Chip, pp. 877-883. 8

Munson, M.S., P. Yager, S.N. Squaw Valley 2003. A Novel Microfluidic Mixer Based on Successive Lamination, 7th Internation Conference on Miniaturized Chemical and Biochemical Analysis Systems, pp. 495-498. 22.

Nguyen, N., Z. Wu 2004. Micromixers - a review, J. of Micromechanics and Microengineering, pp. R1R16.

Ottino, J. M. 1990. Mixing, Chaotic Advection, And Turbulence, Annual Reviews of Fluid Mecahnics, pp. 207-253.

Pust, O., T. Strand, P. Mathys, A. Rutti 2006. Quantification of Laminar Mixing Performance using Laser-Induced Fluorescence, 13th International Symposium on Applications of Laser Techniques to Fluid Mechanics. pp. 26-29.

Park, S., S. Lee, J.H.. Moon, S. Yang 2009. Holographic fabrication of three-dimensional nanostructures for microfluidic passive mixing, Lab on a Chip, pp. 3144-3150. 3.

Rohr, T, Yu C, Davey M H, Svec F, Frechet J M J,. 2001. Porous Polymer Monoliths: Simple and Efficient Mixers Prepared by Direct Polymerization in the Channels of Microfluidic Chips, Electrophoresis, pp. 3959-3967. 89.

Schonfeld, F., V. Hessel, C. Hofmann 2004. An Optimised Split-and-Recombine Micro-Mixer with Uniform 'Chaotic' Mixing., Lab on a Chip, pp. 65-69.

Simonnet, C., A. Groiseman 2005. Chaotic Mixing in a Steady Flow in a Microchannel, Physical Review Letters, pp. 1-4.

Stone, H.A., S. Kim 2001. Microfluidics: Basic Issues, Applications, and Challenges, AIChE Journal, pp. 1250-1254.

Stroock. A.D., S.K.W. Dertinger, A. Ajdari, I. Mezic, H.A. Stone, G.M. Whitesides. 2002. Chaotic Mixer for Microchannels, Science Magazine, Vol. 295, pp. 647-651.

Therriault, D., S.R.. White, J.A. Lewis 2003. Chaotic mixing in three-dimensional microvascular networks fabricated by direct-write assembly, Nature Materials, pp. 265-271.

Vijayendran, R.A, K.M. Motsegood, D.J. Beebe, D.E. Leckband 2002. Evaluation of a ThreeDimensional Micromixer in a Surface-based Biosensor, American Chemical Society, pp. 18241828. 
J. Stevens et al. / JAFM, Vol. 5, No. 4, pp. 91-100, 2012.

Willingham, T.W., C.J. Werth, A.J. Valocchi 2008. Evaluation of the Effects of Porous Media Structure on Mixing-Controlled Reactions Using Pore-Scale Modeling and Micromodel Experiments, Environmental Science and Technology, pp. 3185-3193.

Whitesides, G.M. 2006. The origins and the future of microfluidics, Insight Overview, Vol. 442, pp. 368-373.

Wang, H., W. Li 2007. A Novel 3D Porous Micromixer Fabricated Using Selective Ultrasonic Foaming, $J$. of Micromechanics and Microengineering pp. 1835-1842. 0.

Xiao, Z.Y., A. Wang, J. Perumal, D. Kim 2010. Facile Fabrication of Monolithic 3D Porous Silica Microstructures and a Microfluidic System Embedded with the Microstructure, Advanced Functional Materials, pp. 1473-1479. 0. 